\section{(2) OPEN ACCESS}

\title{
Quantification of pulmonary perfusion in idiopathic pulmonary fibrosis with first pass dynamic contrast- enhanced perfusion MRI
}

\author{
Nicholas D Weatherley (1) 1,2 James A Eaden, ${ }^{1}$ Paul J C Hughes, ${ }^{1}$ Matthew Austin, \\ Laurie Smith 이, ${ }^{1}$ Jody Bray, ${ }^{1}$ Helen Marshall, ${ }^{1}$ Stephen Renshaw, ${ }^{2}$ \\ Stephen M Bianchi, ${ }^{2}$ Jim M Wild ${ }^{1}$
}

\begin{abstract}
- Additional material is published online only. To view, please visit the journal online (http://dx.doi.org/10.1136/ thoraxjnl-2019-214375).
\end{abstract}

${ }^{1}$ Polaris, Imaging group, Dept IICD, University of Sheffield, Sheffield, UK

${ }^{2}$ Academic Directorate of Respiratory Medicine, Sheffield Teaching Hospitals NHS Foundation Trust, Sheffield, UK

\section{Correspondence to} Professor Jim M Wild, Polaris, Imaging group, Dept IICD, The University of Sheffield, Sheffield, UK; j.m.wild@sheffield.ac.uk

Received 28 November 2019 Revised 2 October 2020

Accepted 4 October 2020 Published Online First 3 December 2020

\section{SLinked}

- http://dx.doi.org/10.1136/ thoraxjnl-2020-216382

Check for updates

(C) Author(s) (or their employer(s)) 2021. Re-use permitted under CC BY-NC. No commercial re-use. See rights and permissions. Published by BMJ.

To cite: Weatherley ND, Eaden JA, Hughes PJC, et al. Thorax 2021;76:144-151.

\section{ABSTRACT}

Introduction Idiopathic pulmonary fibrosis (IPF) is a fatal disease of lung scarring. Many patients later develop raised pulmonary vascular pressures, sometimes disproportionate to the interstitial disease. Previous therapeutic approaches that have targeted pulmonary vascular changes have not demonstrated clinical efficacy, and quantitative assessment of regional pulmonary vascular involvement using perfusion imaging may provide a biomarker for further therapeutic insights. Methods We studied 23 participants with IPF, using dynamic contrast-enhanced MRI (DCE-MRI) and pulmonary function tests, including forced vital capacity (FVC), transfer factor $\left(\mathrm{TL}_{\mathrm{CO}}\right)$ and coefficient $\left(\mathrm{K}_{\mathrm{co}}\right)$ of the lungs for carbon monoxide. DCE-MRI parametric maps were generated including the full width at half maximum (FWHM) of the bolus transit time through the lungs. Key metrics used were mean (FWHM ${ }_{\text {mean }}$ ) and heterogeneity $\left(\mathrm{FWHM}_{\mathrm{IOR}}\right)$. Nineteen participants returned at 6 months for repeat assessment.

Results Spearman correlation coefficients were identified between $\mathrm{TL}_{\mathrm{CO}}$ and $\mathrm{FWHM}_{\mathrm{OQR}}(\mathrm{r}=-0.46$; $p=0.026), K_{c o}$ and $F W H M_{\text {mean }}(r=-0.42 ; p=0.047)$ and $\mathrm{K}_{\mathrm{CO}}$ and $\mathrm{FWHM}_{\mathrm{IOR}}(\mathrm{r}=-0.51 ; \mathrm{p}=0.013)$ at baseline. No statistically significant correlations were seen between FVC and DCE-MRI metrics. Follow-up at 6 months demonstrated statistically significant decline in FVC $(p=0.040)$ and $K_{c o}(p=0.014)$, with an increase in $\mathrm{FWHM}_{\text {mean }}(p=0.040)$, but no significant changes in $\mathrm{TL}_{c 0}$ $(p=0.090)$ nor FWHM $(p=0.821)$.

Conclusions DCE-MRI first pass perfusion demonstrates correlations with existing physiological gas exchange metrics, suggesting that capillary perfusion deficit (as well as impaired interstitial diffusion) may contribute to gas exchange limitation in IPF. FWHM showed a significant increase over a 6-month period and has potential as a quantitative biomarker of pulmonary vascular disease progression in IPF.

\section{INTRODUCTION}

Idiopathic pulmonary fibrosis (IPF) is the the most common idiopathic interstitial lung disease, carrying a natural median life expectancy of 2 to 5 years from diagnosis, ${ }^{1}$ although recent advances in antifibrotic therapy may have somewhat improved the outlook. ${ }^{2}$ Most patients with IPF listed for lung transplantation exhibit elevated pulmonary arterial

\section{Key messages}

What is the key question?

- Does dynamic contrast-enhanced MRI (DCEMRI) provides a means of quantifying regional information of pulmonary perfusion in idiopathic pulmonary fibrosis (IPF)?

What is the bottom line?

- DCE-MRI first pass perfusion demonstrates correlations with existing physiological gas exchange metrics, shows increased pulmonary transit times in regions of fibrosis and progression over a 6 -month time period.

Why read on?

- In light of recent trials failing to demonstrate efficacy of pulmonary vasodilator therapy in IPF, DCE-MRI may provide a novel means of phenotyping vascular changes and thus be used to enrich future study populations.

pressures, and many patients in the end stages of disease develop fulminant pulmonary hypertension. ${ }^{3}$ However, more than $60 \%$ of the capillary bed is lost before elevated pulmonary arterial pressures are manifest at the time of right heart catheterisation, ${ }^{4}$ the clinical gold standard of assessment. Recent data from quantitative CT assessment of blood vessel volume density suggests that loss of the small pulmonary vessels may be prognostic in IPF. $^{5}$ Thus, a means of functionally assessing these changes may provide mechanistic insight into lung pathophysiology and a quantitative means of testing interventions targeted at treating the pulmonary vascular changes in IPF at an earlier stage.

Dynamic contrast-enhanced MRI (DCE-MRI) is clinically used for the functional assessment of perfusion, predominantly in imaging the myocardium, brain $^{78}$ and oncological targets. ${ }^{9}{ }^{10}$ Literature for imaging the pulmonary circulation with DCE-MRI in a clinical setting has to date largely focussed on pulmonary vascular disease, ${ }^{11}$ with other studies in disease such as cystic fibrosis and chronic obstructive pulmonary disease generally small and focussed on methodological development. ${ }^{12-14}$

DCE-MRI tracks the passage of a paramagnetic contrast agent bolus applied via a peripheral vein 
through the circulation in the organ of interest. In pulmonary imaging, the contrast can be visualised passing through the right atrium and ventricle, into the pulmonary arterial trunk, the pulmonary circulation, then into the left heart and systemic circulation. From the resultant time-resolved images, qualitative inferences of cardiopulmonary vascular haemodynamic processes such as shunting and bronchial circulation can be observed. Moreover, quantitative metrics of regional blood flow, volume and transit time may be generated, allowing regional and global assessment of pulmonary perfusion to be made.

Pulmonary perfusion metrics have prognostic implications in patients with pulmonary arterial hypertension, ${ }^{11}$ but have undergone little exploration in interstitial lung disease (ILD). There may however be a role for gadolinium-enhanced MRI in the assessment of disease activity. ${ }^{15}$ Tsuchiya et al reported a relationship between reduced pulmonary blood flow measured using phase-contrast MRI and reduced lung volumes in 27 patients with ILD, but did not discover a relationship with lung physiology, perhaps due to the heterogeneity of the lung diseases analysed. ${ }^{16}$ Other studies have identified that delayed or late phase contrast (images taken approximately $10 \mathrm{~min}$ after administration of intravenous gadolinium) mean signal intensity correlates with structural extent of fibrotic lung disease, ${ }^{17}$ and may even evaluate early fibrosis in patients with apparently morphologically normal lung. ${ }^{18}$

Herein, we present first pass perfusion DCE-MRI of the lungs as a means of assessing changes in blood flow through the lung parenchyma of patients with IPF.

\section{METHODS}

\section{Study subjects}

For this prospective, observational study, all participants provided informed written consent. The research was conducted according to the principles of the Declaration of Helsinki. Some participants in this study have been previously reported on in the context of hyperpolarised xenon MRI. ${ }^{19}$ However, none of the DCE perfusion MRI data in this article has been published elsewhere.

Twenty-three participants with a diagnosis of IPF were recruited through a tertiary interstitial lung disease centre. All diagnoses were clarified against international consensus criteria, ${ }^{1}$ in a multidisciplinary setting of respiratory physicians and thoracic radiologists. No lung biopsies were necessary in the diagnostic pathway. Participants had no evidence of pulmonary hypertension at baseline, from transthoracic echocardiography using the Bernoulli equation to assess estimated systolic pulmonary artery pressure from tricuspid jet velocity as a standard method of clinical screening. ${ }^{20}$ Potential participants with stage 4 or 5 chronic kidney disease were excluded prior to enrolment $(n=2)$, due to the reported risk of gadolinium-induced nephrogenic systemic fibrosis in the context of renal failure. ${ }^{21}$

\section{Study design}

Each of the 23 participants underwent a baseline DCE-MRI, between February 2016 and March 2019. Nineteen participants returned for repeat imaging at 6 months (mean 193 days; SD 17.7 days). Four patients withdrew from the study prior to follow-up and subsequently died from respiratory failure. Pulmonary function tests were performed on the same day during both visits.

\section{Imaging and physiology methods}

Images were obtained on a $1.5 \mathrm{~T}$ GE HDx scanner, with an 8-channel thoracic array coil using a three-dimensional spoiled gradient echo time resolved view sharing sequence ${ }^{22}$ with parallel imaging. ${ }^{23}$ Pulse sequence parameters included: voxel size $2.4 \times 6.0 \times 10.0 \mathrm{~mm}^{3}$, bandwidth $250 \mathrm{kHz}$, flip angle $30^{\circ}$, TE $1.1 \mathrm{~ms}$, TR $2.5 \mathrm{~ms}$, frame rate of 2 per s. The imaging temporal resolution ranged from: 0.597 to $0.826 \mathrm{~s}$ per whole lung volume $($ mean $\pm S D=0.664 \pm 0.051 \mathrm{~s})$. Images were taken over $25 \mathrm{~s}$ at the end of tidal breathing following a bolus of $0.05 \mathrm{~mL} \cdot \mathrm{kg}^{-1}$ dose of Gadovist contrast agent (Bayer), injected through a peripherally sited intravenous cannula at a rate of $4 \mathrm{~mL} \cdot \mathrm{s}^{-1}$ with a $20 \mathrm{~mL}$ $0.9 \%$ sodium chloride flush.

Pulmonary function tests included spirometry for forced vital capacity (FVC) and the single breath carbon monoxide assessment of gas exchange in the seated position, providing transfer factor $\left(\mathrm{T}_{\mathrm{LCO}}\right)$ and coefficient $\left(\mathrm{K}_{\mathrm{CO}}\right)$ of the lungs. A $10 \mathrm{~s}$ single breath-hold manoeuvre was used as per international standards ${ }^{24}$ repeated twice to ensure reproducibility.

\section{Analysis}

From the resultant MRI images, full width of half-maximum (FWHM) maps of the first pass of perfusion signal enhancement were derived for each voxel within the lung parenchyma. Each voxel demonstrates an upslope in signal, until a peak signal enhancement is seen and a downslope as contrast exits the voxel. The FWHM (s) is taken as the time taken between the point where $50 \%$ of maximum signal intensity has been reached on the upslope and downslope as represented in the left panel of figure 1. The FWHM was averaged over the whole lung maps to give a mean value $\left(\mathrm{FWHM}_{\text {mean }}\right)$ and the IQR $\left(\mathrm{FWHM}_{\mathrm{IQR}}\right)$ of the distribution of FWHM was used in order to provide a metric of perfusion heterogeneity.

The parametric maps of FWHM were generated for each participant, ${ }^{25}$ performed using custom-written code for MATLAB 2016a (The MathWorks, Inc., Natick, Massachusetts, USA), based on equation (2) from Lin et al. ${ }^{25}$ Each slice was manually segmented from the maximum intensity projection image using ITK-SNAP software. ${ }^{26}$ The left and right pulmonary veins, pulmonary artery and second generation pulmonary arterial vessels were excluded from the segmentation. Voxels were only included in the FWHM calculation a point existed after the peak signal enhancement which was $<50 \%$ of this peak value. Any voxels not analysable were set to zero and excluded from whole lung metrics reported.

All gas transfer metrics are studied in SI units, that is, mmol. $\mathrm{min}^{-1} \cdot \mathrm{kPa}^{-1}$ for $\mathrm{TL}_{\mathrm{CO}}$, or mmol.min ${ }^{-1} \cdot \mathrm{kPa}^{-1} \cdot \mathrm{L}^{-1}$ for $\mathrm{K}_{\mathrm{CO}}$. Per cent predicted values for both $\mathrm{FVC}$ and $\mathrm{TL}_{\mathrm{CO}}$ pertain to the Global Lung Initiative reference equations. ${ }^{27} 28$ Strength of correlations between pulmonary function tests, and MRI metrics were assessed by Spearman's rho. Wilcoxon signed-rank test evaluated the significance of 6-month changes in participants undergoing follow-up. P values stated are two-tailed. Statistical analysis was performed using GraphPad Prism 7 for Mac (GraphPad Software, La Jolla, California, USA).

Post-hoc analyses between participants surviving versus not surviving to 6-month follow-up, as well as participants exceeding versus not exceeding the $95 \%$ upper limit of agreement of reproducibility of FWHM mean $_{\text {over }} 6$ months were undertaken using Mann-Whitney U tests. No correction for multiple testing was performed.

\section{RESULTS}

All examinations were well tolerated, and no adverse events were reported. Baseline demographic, pulmonary function and DCEMRI summary metrics are summarised in table 1. As described 


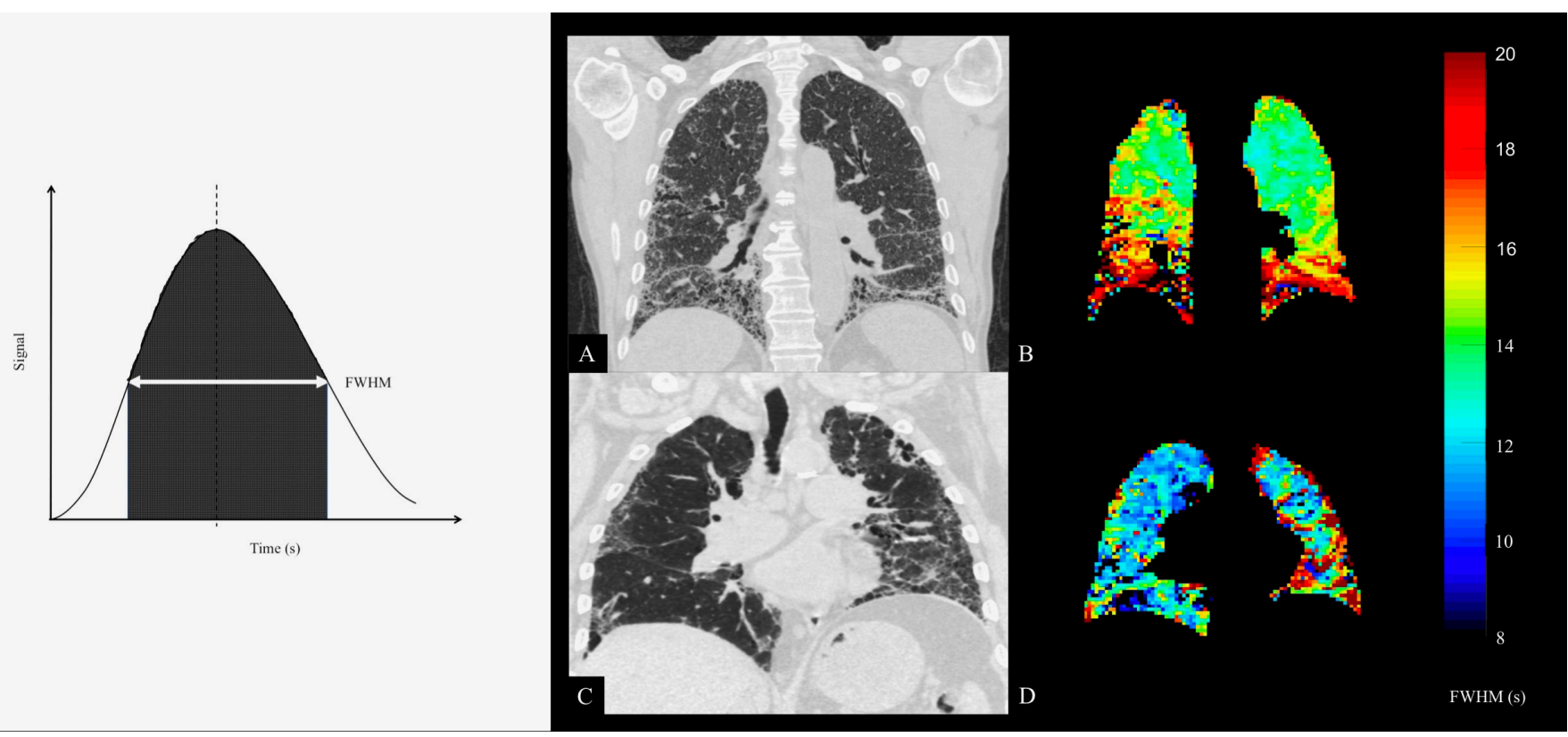

Figure 1 Left panel: Example of signal change through a parenchymal region of interest. The full width at half-maximum (FWHM) signal is often used as a standard threshold for integration. Right panel: CT with best matching coronal slice mean transit time (FWHM) maps derived from dynamic contrast-enhanced MRI from two participants $(A+B$ and $C+D)$. Reticulation and honeycombing on the $C T$ is related to regions of increased transit time in basal and peripheral regions. Colour bar denotes FWHM in seconds.

above, any voxels not analysable were set to zero and excluded from whole lung metrics reported. This resulted in (mean $\pm S D$ ) $80.5 \% \pm 13.6 \%$ of voxels being included over the cohort. Details for each subject are available in the online supplemental material.

\section{Reproducibility}

During the first study visit, a cohort of six subjects underwent repeat DCE-MRI approximately 2 hours apart. Mean interscan bias in $\mathrm{FWHM}_{\text {mean }}$ was $0.35 \mathrm{~s}$ (95\% CI -0.95 to $\left.1.66 \mathrm{~s}\right)$, while mean interscan bias in $\mathrm{FWHM}_{\mathrm{IQR}}$ was $1.05 \mathrm{~s}(95 \% \mathrm{CI}-3.62$ to $5.74 \mathrm{~s})$. Bland-Altman plots of repeatability can be found in the online supplement. An example quantitative parametric map of a subject undergoing imaging twice at baseline is provided in figure 2 .

Table 1 Participant characteristics and baseline data

\begin{tabular}{|c|c|c|}
\hline Demographic & Median & IQR \\
\hline Age (years) & 69.5 & 65.7 to 71.6 \\
\hline Sex & 19 male; 4 female & \\
\hline Height (cm) & 171 & 169 to 178 \\
\hline \multicolumn{3}{|l|}{ Pulmonary function test } \\
\hline FVC (\% predicted) & 83.1 & 70.9 to 95.7 \\
\hline $\mathrm{TL}_{\mathrm{co}}(\%$ predicted $)$ & 54.1 & 40.5 to 77.0 \\
\hline $\mathrm{K}_{\mathrm{co}}(\%$ predicted $)$ & 83.1 & 68.1 to 92.1 \\
\hline \multicolumn{3}{|l|}{ DCE-MRI metrics } \\
\hline $\mathrm{FWHM}_{\text {mean }}(\mathrm{s})$ & 8.01 & 7.01 to 8.88 \\
\hline $\mathrm{FWHM}_{\mathrm{IOR}}(\mathrm{s})$ & 3.66 & 2.72 to 6.51 \\
\hline
\end{tabular}

DCE-MRI, dynamic-contrast enhanced MRI; FVC, forced vital capacity; FWHM, full width at half maximum; FWHM $_{\mathrm{IQR}^{\prime}}$ IQR of the FWHM; FWHM ${ }_{\text {mean }}$, mean FWHM; $\mathrm{K}_{\mathrm{CO} 0^{\prime}}$ diffusing coefficient of the lungs for carbon monoxide; $\mathrm{TL}_{\mathrm{co}^{\prime}}$ diffusing capacity of the lungs for carbon monoxide.

\section{Correlation with baseline pulmonary function tests}

No correlation was found between FVC and FWHM $(\mathrm{r}=-0.22 ; \mathrm{p}=0.310 ; 95 \% \mathrm{CI}-0.59$ to 0.22$)$, or $\mathrm{FVC}$ and FWHM $_{\text {IQR }}(\mathrm{r}=-0.14 ; \mathrm{p}=0.529 ; 95 \% \mathrm{CI}-0.53$ to 0.30$)$. However, a mild strength of correlation trends were found between $\mathrm{TL}_{\mathrm{CO}}$ and $\mathrm{FWHM}_{\text {mean }}(\mathrm{r}=-0.41 ; \mathrm{p}=0.054 ; 95 \%$ CI -0.71 to 0.02$)$ and also $\mathrm{TL}_{\mathrm{CO}}$ and $\mathrm{FWHM}_{\mathrm{IOR}}(\mathrm{r}=-0.46$; $\mathrm{p}=0.026 ; 95 \% \mathrm{CI}-0.74$ to -0.05$)$. $\mathrm{K}_{\mathrm{CO}}$ showed mild-tomoderate correlations with both $\mathrm{FWHM}_{\text {mean }}(\mathrm{r}=-0.42$; $\mathrm{p}=0.047 ; 95 \% \mathrm{CI}-0.71$ to 0.01$)$ and $\mathrm{FWHM}_{\mathrm{IQR}}(\mathrm{r}=-0.51$; $\mathrm{p}=0.01395 \% \mathrm{CI}-0.77$ to -0.11$)$. There was a strong correlation between the two MRI metrics, FWHM $\mathrm{Fman}_{\text {mean }}$ and FWM $\mathrm{FQR}_{\mathrm{IQR}}$ $(\mathrm{r}=0.77 ; \mathrm{p}<0.001 ; 95 \%$ CI 0.52 to 0.90$)$.

\section{Change over 6 months}

Figure 3 demonstrates changes in $\mathrm{FVC}, \mathrm{TL}_{\mathrm{CO}}, \mathrm{FWHM}_{\text {mean }}$ and FWHM $_{\text {IQR }}$ over 6 months in the 19 patients followed up to 6 months. FVC decreased by a median of $2.7 \%(p=0.040$; estimated $95 \% \mathrm{CI}-6.0 \%$ to $1.02 \%)$. $\mathrm{TL}_{\mathrm{CO}}$ decreased by a median of $0.05 \mathrm{mmol} \cdot \mathrm{min}^{-1} \cdot \mathrm{kPa}^{-1}(2.9 \%)(\mathrm{p}=0.090$; estimated $95 \% \mathrm{CI}$ $-4.86 \%$ to $1.02 \%)$. $\mathrm{K}_{\mathrm{CO}}$ decreased by a median of $0.03 \mathrm{mmol}$. $\min ^{-1} \cdot \mathrm{kPa}^{-1} \cdot \mathrm{L}^{-1} \quad(\mathrm{p}=0.014$; estimated $95 \% \mathrm{CI}-0.06$ to 0.00 mmol. $\left.\mathrm{min}^{-1} \cdot \mathrm{kPa}^{-1} \cdot \mathrm{L}^{-1}\right)$. FWHM $\mathrm{FWan}_{\text {mean }}$ increased by a median of $1.05 \mathrm{~s}(\mathrm{p}=0.040$; estimated $95 \% \mathrm{CI}-0.65$ to $3.14 \mathrm{~s})$, whereas FWHM $_{\mathrm{IQR}}$ increased by a median of $0.09 \mathrm{~s}$, but was not significant ( $p=0.821$; estimated $95 \% \mathrm{CI}-1.87$ to $0.95 \mathrm{~s})$.

There were no statistically significant correlations between 6-month change in any of the pulmonary function tests and $\mathrm{FWHM}_{\text {mean }}$, nor FWHM $\mathrm{FR}_{\mathrm{IOR}}$. There was a correlation between 6-month change in FWHM ${ }_{\text {mean }}$ and 6-month change in FWHM $\mathrm{IQR}_{\mathrm{I}}$ $(\mathrm{r}=0.55 ; \mathrm{p}=0.015)$. All correlations are summarised in table 2 .

\section{Qualitative imaging analysis}

The right panel of figure 1 shows the parametric maps of FWHM to demonstrate the spatial relationship between regions 


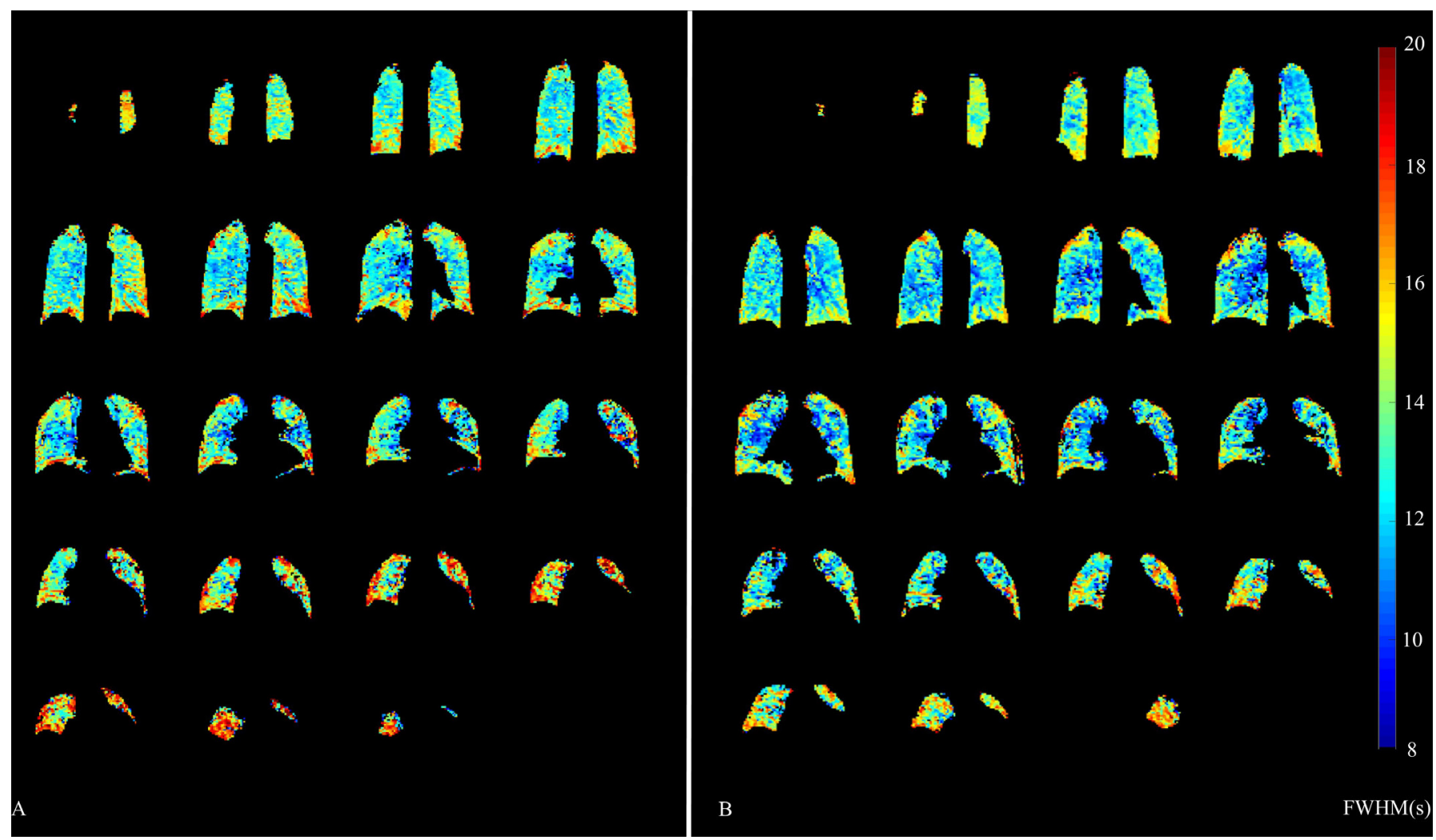

Figure 2 Reproducibility of regional full width at half maximum (FWHM). An example of one subject undergoing same day repeat imaging at two time points ( $A$ and $B$ ). Colour bar denotes FWHM in seconds.

of increased blood transit time and presence of fibrotic lung disease on the accompanying CT coronal image of the same participant. Figure 4 demonstrates progression of transit time across a 6-month time period in two participants.

\section{Post-hoc analysis of subjects exceeding the FWHM limit of reproducibility}

Exploratory analyses were performed on those participants in whom the 6-month increase in FWHM mean $_{\text {exceeded the }}$ upper limit of agreement on the Bland-Altman analysis (change $>1.66 \mathrm{~s}$ ) and those who did not meet this threshold (change $\leq 1.66 \mathrm{~s}$ ). Herein, these groups are referred to as progressors/ non-progressors, respectively, for clarity.

Baseline FWHM (median difference $-0.73 \mathrm{~s}$; 95\% CI -1.55 to $0.95 \mathrm{~s})$ and baseline FVC $(-2.22 \%$; 95\% CI -14.9

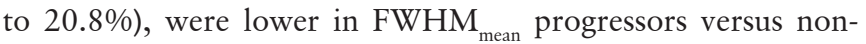
progressors. Baseline $\mathrm{TL}_{\mathrm{CO}}$ (median difference $20.8 \%$; 95\% CI -16.6 to $34.4 \%$ ) and change in $\mathrm{TL}_{\mathrm{CO}}$ (median difference $4.4 \%$; $95 \% \mathrm{CI}-5.15$ to $5.73 \%$ ) were higher in $\mathrm{FWHM}_{\text {mean }}$ progressors versus non-progressors. None of these differences were statistically significant.

\section{Post-hoc analysis of participant survival}

Mean FWHM mean $_{\text {and FWHM }}$ aQR were compared in a post-hoc analysis between participants surviving to 6-month follow-up $(n=19)$ and non-survivors $(n=4)$. Both the FWHM mean $_{\text {and }}$ FWHM $_{\mathrm{IQR}}$ were on average higher in the non-survivors $(1.10 \mathrm{~s}$ and $1.29 \mathrm{~s}$, respectively), although neither was statistically significant $(\mathrm{p}=0.218 ; 95 \% \mathrm{CI}-0.77$ to 3.71 and $\mathrm{p}=0.785 ; 95 \% \mathrm{CI}$ -1.93 to 6.66 , respectively). The small sample size in the nonsurvivor group should be noted.

\section{DISCUSSION}

The transit time of contrast through the lung increases in proportion to worsening gas exchange in our IPF cohort and exhibits a mild strength of non-parametric correlation with established metrics of gas exchange, but not FVC. The FWHM mean $_{\text {of the }}$ transit time demonstrates an increase in our sample IPF population over a 6-month follow-up period and the IQR $\left(\mathrm{FWHM}_{\mathrm{IQR}}\right)$ may be a simple means of describing perfusion heterogeneity. Regional increases in transit time, although analysed on a purely qualitative basis in this study, appear to be spatially congruous with the known peripheral and basal distribution of disease in IPF.

Previous literature suggests changes in lung perfusion may represent loss of the vascular bed due to vascular remodelling. ${ }^{29}$ Histologically, fibrotic regions contain epithelial cells undergoing apoptosis, but also proliferation, ${ }^{30} 31$ leading to a complex and aberrant vascular network involving anastomoses between alveolar capillaries and pulmonary veins. This disruption of perfusion contributes along with alveolar destruction and septal thickening to gas exchange impairment and thus explain the correlation we see between $\mathrm{FWHM}_{\mathrm{IQR}}$ and $\mathrm{TL}_{\mathrm{CO}}$. Patients with IPF are at increased risk of venous and arterial clot formation, ${ }^{32}$ leading to suggestions that intramural thrombus in the pulmonary vasculature may also contribute to perfusion limitation in IPF.

Our model assumes that the FWHM mean is a surrogate of transit time of blood through the lung and therefore can represent blood flow; with the FWHM ${ }_{\mathrm{IQR}}$ a measure of heterogeneity. ${ }^{11} 33$ IPF is generally thought of as a disease of the lung parenchyma, rather than a disease of perfusion limitation and while the parenchymal disease leading to diffusion limitation is the significant 

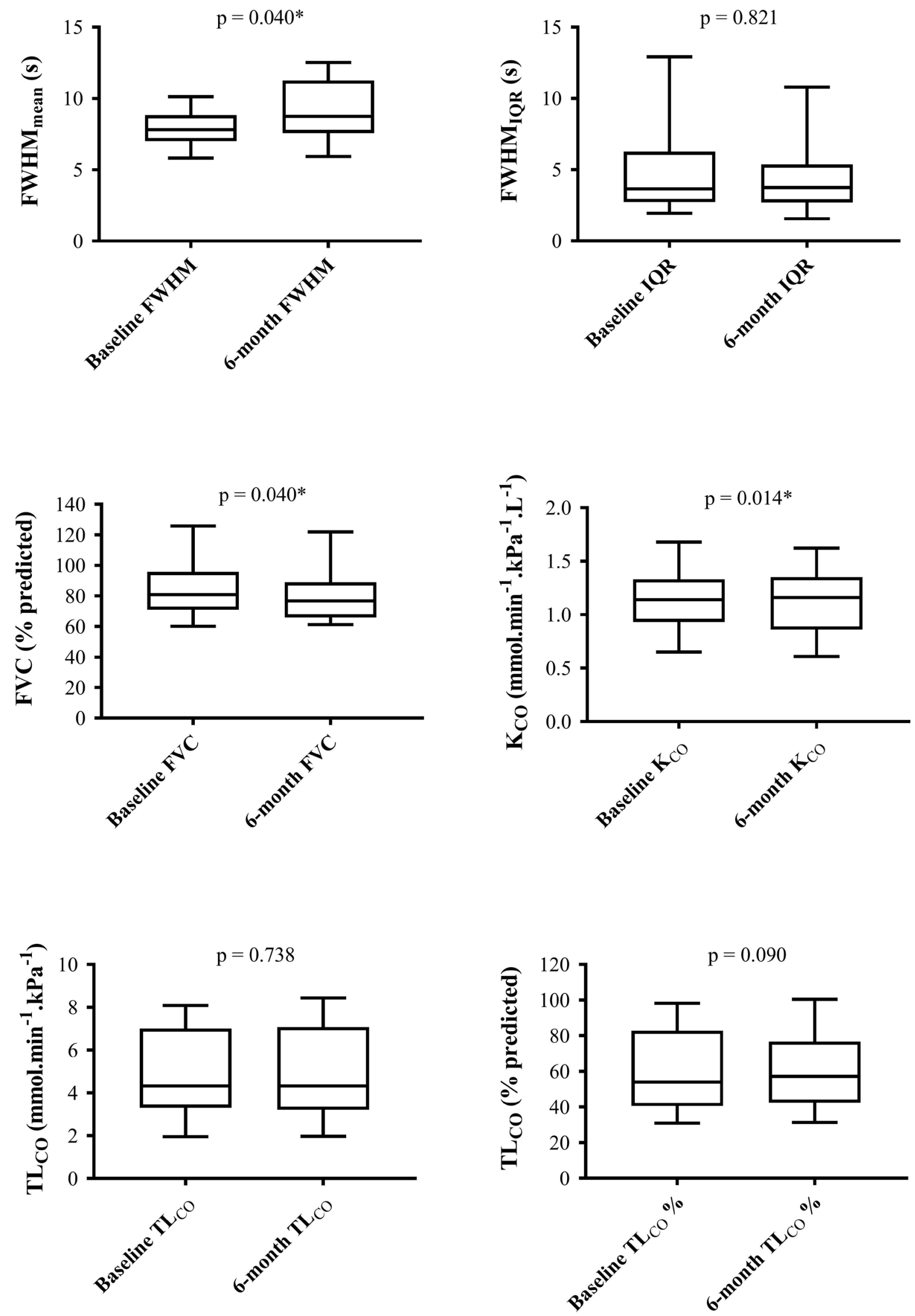

Figure 3 Box and whisker diagrams of baseline and 6-month changes in $\mathrm{FWHM}_{\text {mean }}(\mathrm{A}) ; \mathrm{FWHM}_{\mathrm{IQR}}(\mathrm{B}) ; \mathrm{FVC} \%$ predicted $(\mathrm{C}) ; \mathrm{K}_{\mathrm{CO}} \mathrm{SI}$ units $(\mathrm{D}) ; \mathrm{TL}_{\mathrm{CO}} \mathrm{SI}$ units (E); $\mathrm{TL}_{\mathrm{CO}} \%$ predicted (). Wilcoxon signed-rank $\mathrm{p}$ values quoted for each. FVC, forced vital capacity; FWHM, full width at half maximum; FWHM ${ }_{10 R^{\prime}}$ IQRof the FWHM; FWHM ${ }_{\text {mean' }}$ mean $\mathrm{FWHM;} \mathrm{K}_{\mathrm{CO} \text {, }}$ diffusingcoefficient of the lungs for carbon monoxide; $\mathrm{TL}_{\mathrm{CO}{ }^{\prime}}$ diffusingcapacity of the lungs for carbon monoxide. 
Table 2 Summary of correlations between pulmonary function tests and MRI metrics

\begin{tabular}{|c|c|c|c|c|c|c|}
\hline \multirow[b]{2}{*}{ Pulmonary function test } & \multicolumn{3}{|c|}{$\mathrm{FWHM}_{\text {mean }}$} & \multicolumn{3}{|c|}{ FWHM $_{\text {IQR }}$} \\
\hline & $r$ & $P$ value & $95 \% \mathrm{Cl}$ & $r$ & $P$ value & $95 \% \mathrm{Cl}$ \\
\hline FVC & -0.22 & 0.310 & -0.59 to 0.22 & -0.14 & 0.529 & -0.53 to 0.30 \\
\hline $\mathrm{TL}_{\mathrm{co}}$ & -0.41 & 0.054 & -0.71 to 0.02 & -0.46 & $0.026^{*}$ & -0.74 to 0.05 \\
\hline \multirow[t]{2}{*}{$\mathrm{K}_{\mathrm{co}}$} & -0.42 & $0.047^{*}$ & -0.71 to 0.01 & -0.51 & $0.013^{*}$ & -0.77 to 0.11 \\
\hline & \multicolumn{3}{|c|}{ Change in $\mathrm{FWHM}_{\text {mean }}$} & \multicolumn{3}{|c|}{ Change in $\mathrm{FWHM}_{\mathrm{IQR}}$} \\
\hline 6-month change in FVC & 0.02 & 0.949 & -0.45 to 0.48 & -0.34 & 0.154 & -0.70 to 0.15 \\
\hline 6-month change in $\mathrm{TL}_{\mathrm{co}}$ & 0.16 & 0.537 & -0.35 to 0.59 & 0.07 & 0.791 & -0.43 to 0.53 \\
\hline 6-month change in $\mathrm{K}_{\mathrm{co}}$ & -0.39 & 0.095 & -0.72 to 0.07 & -0.10 & 0.695 & -0.54 to 0.39 \\
\hline
\end{tabular}

${ }^{*}$ Designates $p$-value $<0.05$

FVC, forced vital capacity; FWHM, full width at half maximum; FWHM ${ }_{I Q R^{\prime}}$ IQR of the FWHM; FWHM mean' mean FWHM; $\mathrm{K}_{\mathrm{CO}^{\prime}}$ diffusing coefficient of the lungs for carbon monoxide; $\mathrm{TL}_{\mathrm{co}}$ diffusing capacity of the lungs for carbon monoxide.

mechanism that causes changes in $\mathrm{TL}_{\mathrm{CO}}$, the accompanying changes in capillary perfusion should also be considered in the understanding of gas exchange in IPF. If DCE-MRI can indeed identify subclinical changes in pulmonary perfusion, this technology may fulfil a clinical need to identify patients with early pulmonary vascular changes, disproportionate to the degree of ILD. Anecdotal observations of clinical improvement of some patients with IPF on sildenafil have been reported and there is biological plausibility of its effect, ${ }^{34}$ even though a randomised controlled trial of patients with IPF and low $\mathrm{TL}_{\mathrm{CO}}$ demonstrated no significant difference between subjects on sildenafil or placebo. ${ }^{35}$

Our findings differ somewhat from the findings of Tsuchiya et al, who used phase contrast MRI to evaluate blood flow within the pulmonary arteries. ${ }^{16}$ They found no relationship between pulmonary arterial flow and diffusing capacity, although severity quantified by CT was correlated with pulmonary arterial flow. ${ }^{16}$ Their cohort was a wider population of patients with ILD, some of whom had IPF. Our population had a greater degree of diffusion limitation (although the carbon monoxide gas diffusion coefficient, $\mathrm{K}_{\mathrm{CO}}$ is not reported in their data) and a relatively better preserved FVC. Moreover, measurement of blood flow in the major pulmonary arteries is a global measurement of pulmonary vascular resistance and while affected by the downstream changes in microvascular blood flow is not a direct measure of capillary perfusion.

Given that much of the capillary bed is lost before elevated pulmonary artery pressures are manifest, a sensitive means of detecting vascular changes earlier in the process may allow clinical trial stratification for those at risk of pulmonary hypertension, or better inform lung transplantation decisions. Such markers may complement other MR sequences used in the

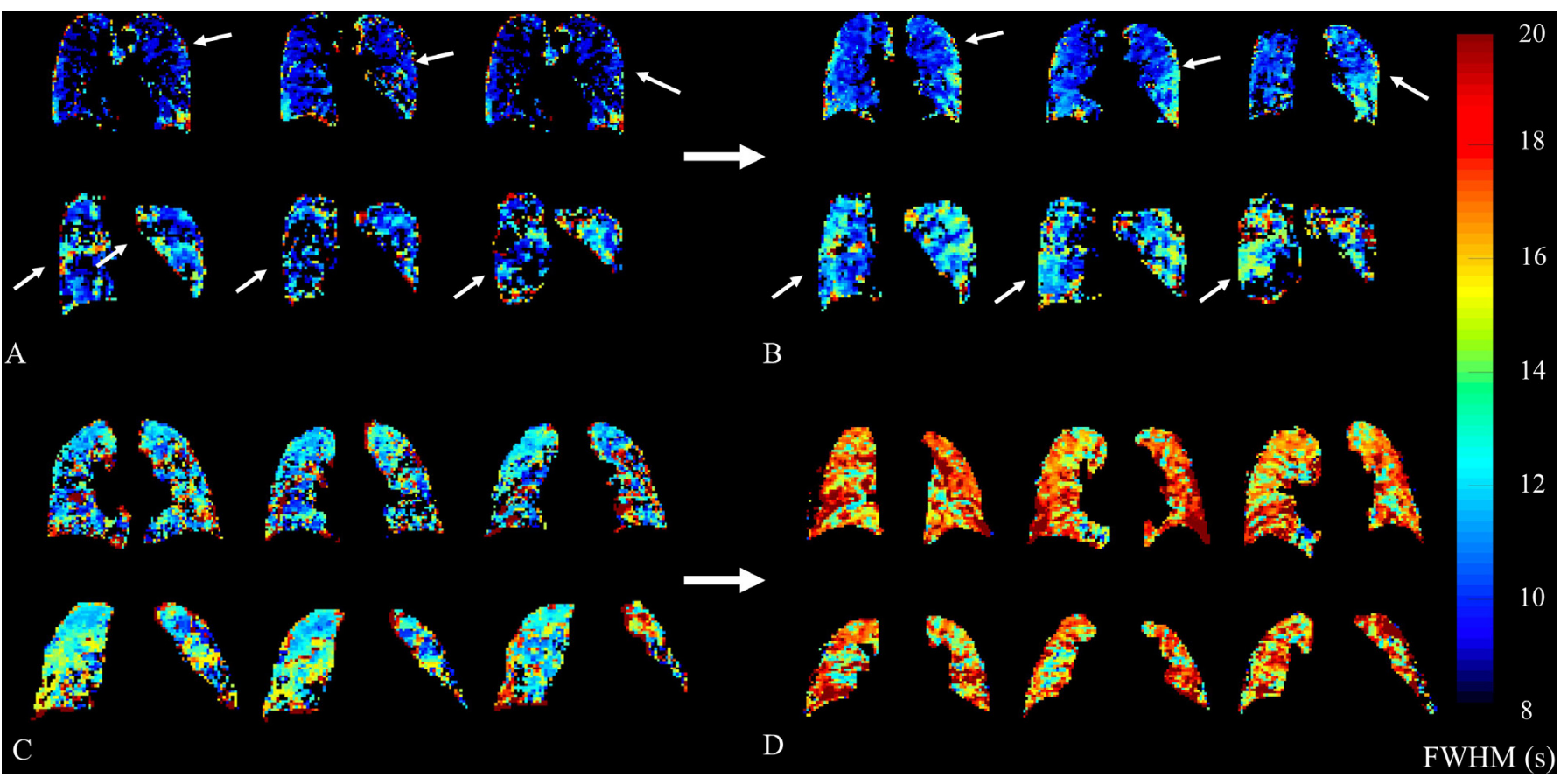

Figure 4 Representative parametric transit time (FWHM) maps from two participants each with baseline (A and C) and follow up (B and D) scans. Participant 12 ( $A$ and $B$ ) demonstrates an increase in FWHM in the right lower lobe and left mid-zone peripheral lung tissue. FWHM mean $_{\text {decreased }}$ slightly by $0.13 \mathrm{~s}$, with associated changes in FVC from $60.1 \%$ to $63.2 \%$, and $\mathrm{TL}_{\mathrm{co}}$ from $37.1 \%$ to $33.0 \%$, respectively. Participant 18 (C and D) shows a gross overall increase in FWHM, again worse in peripheral lung tissue. $\mathrm{FWHM}_{\text {mean }}$ increased by $4.35 \mathrm{~s}$, in spite of both $\mathrm{FVC}_{\text {and }} \mathrm{TL}_{\mathrm{co}}$ remaining relatively stable from $76.0 \%$ to $74.5 \%$, and from $87.2 \%$ to $88.2 \%$, respectively. Colour bar denotes FWHM in seconds. FVC, forced vital capacity; FWHM, full width at half maximum; $\mathrm{FWHM}_{\text {mean' }}$ mean $\mathrm{FWHM} \mathrm{TL}_{\mathrm{CO}^{\prime}}$ diffusingcapacity of the lungs for carbon monoxide. 
assessment of ILD. For example, ultrashort echo time MRI demonstrates improved structural image quality and resolution compared with conventional sequences and also provides a nonionising alternative to CT and hyperpolarised xenon MRI has the potential to provide longitudinal, ${ }^{19}$ regional $^{36}$ metrics of gas exchange.

The use of global measures is however, potentially problematic. It is possible that the increase in FWHM ${ }_{\text {mean }}$ over time is representative of progression of another aspect of the disease process, rather than vascular injury. However, the participants with and apparently progressive $\mathrm{FWMH}_{\text {mean }}$ have a higher baseline $\mathrm{TL}_{\mathrm{CO}}$ and do not appear more likely to demonstrate progression in $\mathrm{TL}_{\mathrm{CO}}$, nor FVC. Qualitatively, regions of increased FWHM appear analogous with regions of fibrotic disease on CT, though no quantitative analysis yet exists to support this. Therefore, assessment of the regional correlation of perfusion maps with ${ }^{129} \mathrm{Xe}$ maps of tissue and red blood cell uptake, alongside quantitative regional assessment of CT is a logical next step in regional understanding of gas exchange using multimodal MRI.

The main limitation of this study is that it was conducted in a single-centre and in a relatively small number of participants. However, the statistically significant change seen in FWHMat 6 months demonstrates the potential of this approach for assessment of disease progression. Future work to provide description of more quantitative regional changes in other perfusion parameters may provide a means of detecting gradual progression. From a DCE methodological perspective, ongoing work with full quantitative perfusion analysis is being pursued in order to generate associated maps of regional blood flow and blood volume using T1 mapping and arterial input function deconvolution. ${ }^{37-39}$

Consideration should also be given to the role of anatomical positioning on perfusion. While $\mathrm{TL}_{\mathrm{CO}}$ is typically measured in the sitting position, MRI is performed supine. This leads to a redistribution of flow from the usually dependant and optimally perfused basal lung tissue to the rest of the lung, which could affect transit times in the bases. However, one would still not expect transit times to be slower in the lung bases than the rest of the lung tissue in health in the supine position as is clearly the case in the example parametric maps provided. This makes the observed qualitative findings of increased basal and peripheral transit time much more likely to be disease related. As is visible in figure 2, there is greater repeated-measurement disagreement in the anterior slices. We propose this is likely due to the presence an anterior-posterior perfusion gradient, caused by blood pooling towards the posterior thorax under gravitational effect while the subject is in a supine position. This reduces the signal from the anterior slices and therefore reduces the signal to noise ratio. The anterior slices also contain a smaller volume of lung tissue, greater increasing the margin of error. The postural gradient and its associated impact on the findings should be considered in future studies.

The participants in our study did not undergo right heart catheterisation, the gold standard assessment for the presence of pulmonary hypertension. However, echocardiography is sensitive in detecting the presence of pulmonary hypertension and is clinically used as a screening tool, ${ }^{40}$ and as such best represents the investigation that is routinely available in standard clinical practice.

Debate exists around the best technique by which to control for the effects of the respiratory cycle and thus intrathoracic pressure effects on pulmonary perfusion. Here, we have chosen end of tidal breathing to facilitate breath holding and reduce the risk of a Valsalva manoeuvre being performed. Respiratory gating may ensure functional residual volume is met, but is not available in many centres and regardless, the measurement of first pass enhancement requires the participant to perform a breath holding technique to mitigate for the effects of variation in intrathoracic pressures. Further methodological work on the optimal breath-hold technique may therefore be of value in future studies. Interscan reproducibility results may also be affected by slight alteration of the temporal resolution for each case. This was due to the bespoke slice positioning by the MR radiographer on each scan session. This as an experimental limitation that could be avoided with more stringent control of the slice positioning in future work. One possible solution would be to fix the slice coverage to ensure the same inter frame rate between all subjects. Further details of the temporal resolution changes in the six participants who underwent same-day reproducibility imaging are presented in the online supplemental table. One significant consideration is the role of cardiac function in the quantitative metrics of our analysis. While patients with significant cardiac dysfunction were not recruited to this study, in clinical practice, the cardiac function of sexagenarian and septuagenarian population is likely to vary. This may in turn cause changes in global pulmonary flow metrics. Both systolic and diastolic dysfunction are commonly comorbid conditions in this age bracket and future studies may wish to consider including measures of systolic and diastolic cardiac function, either in the form of transthoracic echocardiography, or possibly in the same imaging sitting as the investigative endpoint with cardiac MRI.

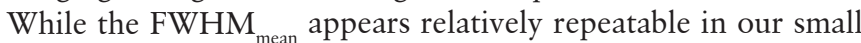
cohort, the FWHM ${ }_{\mathrm{IQR}}$ has a significant degree of variability, which may be problematic to its use as a clinical biomarker.

Overall, $80 \%$ of voxels returned an analysable result, but this leaves a not insignificant amount of the lung tissue that could not be analysed. The overall reduced perfusion in the IPF lung may induce underestimation bias of the transit time, particularly in the most severely affected disease. Future work on developing the methodology of this approach therefore needs to account for this.

The potential effects of antifibrotic therapy are not accounted for in this study, and some participants commenced antifibrotic therapeutics after the initial baseline study visit, which may therefore affect follow-up results. However, none of these participants were taking pulmonary vasodilator therapy and none were part of the recently reported trial of nintedanib/sildenafil versus nintedanib/placebo. ${ }^{35}$

In conclusion, DCE lung perfusion MRI is feasible and welltolerated in a population of participants with IPF. Of the two candidate markers described here, $\mathrm{FWHM}_{\text {mean }}$ appears to be more reproducible than $\mathrm{FWHM}_{\mathrm{IOR}}$. Although there is a paucity of data to determine reliably whether the mean interscan bias of $0.35 \mathrm{~s}$ is significant, it appears lower than the mean 6-month change of $1.05 \mathrm{~s}$. Whole lung metrics of transit time correlate to existing metrics of perfusion-limited gas $\left(\mathrm{TL}_{\mathrm{CO}}\right)$ and $\mathrm{FWHM}_{\mathrm{m}}$ significantly increases over a 6 -month time interval. The regional information contained within the parametric maps may provide a novel means of assessing early perfusion changes in this population in the absence of detectable pulmonary hypertension on echocardiography and future research in this area could focus on quantifying these changes on a regional basis to enhance the sensitivity of this technique for use in early intervention trials.

Twitter Stephen Renshaw @steverenshaw

Acknowledgements The authors would like to acknowledge the administrative support provided to this study by Leanne Armstrong and Jennifer Rodgers at POLARIS, University of Sheffield, UK. We also thank the National Institute of Health 
Research (NIHR-RP-R3-12-027), Medical Research Council (MR/M008894/1) and GlaxoSmithKline (BIDS3000032592) for funding.

Contributors Conceptualisation: NDW, PH, SR, SMB, JMW; Methodology: NDW, SR, SMB, JMW; Software: JB, HM, JMW; Formal Analysis: NDW, JAE, PH, HM; Investigation: NDW, JAE, MA, LS, JB; Wrting - original draft: NDW; Writing - review and editing: All authors; Supervision: SR, SMB, JMW; Project Administration: NDW Funding Acquisition: JMW.

Funding This study is funded by the National Institute of Health Research (NIHRRP-R3-12-027), Medical Research Council (MR/M008894/1) and GlaxoSmithKline (BIDS3000032592).

Competing interests None declared.

Patient consent for publication Not required.

Ethics approval Ethical approval was given by North West NHS Research Ethics Committee.

Provenance and peer review Not commissioned; externally peer reviewed.

Data availability statement All data relevant to the study are included in the article or uploaded as supplementary information.

Open access This is an open access article distributed in accordance with the Creative Commons Attribution Non Commercial (CC BY-NC 4.0) license, which permits others to distribute, remix, adapt, build upon this work non-commercially, and license their derivative works on different terms, provided the original work is properly cited, appropriate credit is given, any changes made indicated, and the use is non-commercial. See: http://creativecommons.org/licenses/by-nc/4.0/.

ORCID iDs

Nicholas D Weatherley http://orcid.org/0000-0001-5589-2219

Laurie Smith http://orcid.org/0000-0002-5769-423X

\section{REFERENCES}

1 Raghu G, Collard HR, Egan JJ, et al. An official ATS/ERS/JRS/ALAT statement: idiopathic pulmonary fibrosis: evidence-based guidelines for diagnosis and management. Am J Respir Crit Care Med 2011:183:788-824.

2 Nathan SD, Albera C, Bradford WZ, et al. Effect of continued treatment with pirfenidone following clinically meaningful declines in forced vital capacity: analysis of data from three phase 3 trials in patients with idiopathic pulmonary fibrosis. Thorax 2016:71:429-35.

3 Nathan SD, Shlobin OA, Ahmad S, et al. Serial development of pulmonary hypertension in patients with idiopathic pulmonary fibrosis. Respiration 2008;76:288-94.

4 Wright JL, Churg A. Effect of long-term cigarette smoke exposure on pulmonary vascular structure and function in the guinea pig. Exp Lung Res 1991;17:997-1009

5 Jacob J, Bartholmai BJ, Rajagopalan S, et al. Mortality prediction in idiopathic pulmonary fibrosis: evaluation of computer-based CT analysis with conventional severity measures. Eur Respir J 2017:49:1601011.

6 Panting JR, Gatehouse PD, Yang G-Z, et al. Abnormal subendocardial perfusion in cardiac syndrome $X$ detected by cardiovascular magnetic resonance imaging. $N$ Engl f Med 2002:346:1948-53.

7 MacDonald ME, Smith MR, Frayne R. Deconvolution with simple extrapolation for improved cerebral blood flow measurement in dynamic susceptibility contras magnetic resonance imaging during acute ischemic stroke. Magn Reson Imaging 2011;29:620-9.

8 Wang DJJ, Alger JR, Qiao JX, et al. The value of arterial spin-labeled perfusion imaging in acute ischemic stroke: comparison with dynamic susceptibility contrast-enhanced MRI. Stroke 2012;43:1018-24.

9 Preziosi P, Orlacchio A, Di Giambattista G, et al. Enhancement patterns of prostate cancer in dynamic MRI. Eur Radiol 2003;13:925-30.

10 Leach MO, Brindle KM, Evelhoch JL, et al. The assessment of antiangiogenic and antivascular therapies in early-stage clinical trials using magnetic resonance imaging: issues and recommendations. Br J Cancer 2005;92:1599-610.

11 Swift AJ, Telfer A, Rajaram S, et al. Dynamic contrast-enhanced magnetic resonance imaging in patients with pulmonary arterial hypertension. Pulm Circ 2014:4:61-70.

12 Salehi Ravesh M, Brix G, Laun FB, et al. Quantification of pulmonary microcirculation by dynamic contrast-enhanced magnetic resonance imaging: comparison of four regularization methods. Magn Reson Med 2013;69:188-99.

13 Ingrisch M, Maxien D, Schwab F, et al. Assessment of pulmonary perfusion with breath-hold and free-breathing dynamic contrast-enhanced magnetic resonance imaging: quantification and reproducibility. Invest Radiol 2014;49:382-9.
14 Pienn M, Kovacs G, Tscherner M, et al. Non-Invasive determination of pulmonary hypertension with dynamic contrast-enhanced computed tomography: a pilot study. Eur Radiol 2014;24:668-76.

15 Ley S, Ley-Zaporozhan J. Pulmonary perfusion imaging using MRI: clinical application. Insights Imaging 2012;3:61-71.

16 Tsuchiya N, Yamashiro T, Murayama S. Decrease of pulmonary blood flow detected by phase contrast MRI is correlated with a decrease in lung volume and increase of lung fibrosis area determined by computed tomography in interstitial lung disease. Eur J Radiol 2016;85:1581-5.

17 Lavelle LP, Brady D, McEvoy S, et al. Pulmonary fibrosis: tissue characterization using late-enhanced MRI compared with unenhanced anatomic high-resolution CT. Diagn Interv Radiol 2017;23:106-11.

18 Mirsadraee S, Tse M, Kershaw L, et al. T1 characteristics of interstitial pulmonary fibrosis on 3T MRI-a predictor of early interstitial change? Quant Imaging Med Surg 2016;6:42-9.

19 Weatherley ND, Stewart NJ, Chan H-F, et al. Hyperpolarised xenon magnetic resonance spectroscopy for the longitudinal assessment of changes in gas diffusion in IPF. Thorax 2019;74:500-2.

20 Fisher MR, Forfia PR, Chamera E, et al. Accuracy of Doppler echocardiography in the hemodynamic assessment of pulmonary hypertension. Am J Respir Crit Care Med 2009;179:615-21.

21 Khawaja AZ, Cassidy DB, Al Shakarchi J, et al. Revisiting the risks of MRI with gadolinium based contrast agents-review of literature and guidelines. Insights Imaging 2015:6:553-8.

22 Korosec FR, Frayne R, Grist TM, et al. Time-Resolved contrast-enhanced 3D Mr angiography. Magn Reson Med 1996;36:345-51.

23 Pruessmann KP, Weiger M, Scheidegger MB, et al. Sense: sensitivity encoding for fast MRI. Magn Reson Med 1999:42:952-62.

24 Graham BL, Brusasco V, Burgos F, et al. 2017 ERS/ATS standards for single-breath carbon monoxide uptake in the lung. Eur Respir J 2017;49:1600016.

25 Lin Y-R, Tsai S-Y, Huang T-Y, et al. Inflow-weighted pulmonary perfusion: comparison between dynamic contrast-enhanced MRI versus perfusion scintigraphy in complex pulmonary circulation. J Cardiovasc Magn Reson 2013;15:21.

26 Yushkevich PA, Piven J, Hazlett HC, et al. User-guided 3D active contour segmentation of anatomical structures: significantly improved efficiency and reliability. Neuroimage 2006;31:1116-28

27 Quanjer PH, Stanojevic S, Cole TJ, et al. Multi-Ethnic reference values for spirometry for the 3-95-yr age range: the global lung function 2012 equations. Eur Respir J 2012:40:1324-43.

28 Stanojevic S, Graham BL, Cooper BG, et al. Official ERS technical standards: global lung function initiative reference values for the carbon monoxide transfer factor for Caucasians. Eur Respir J 2017;50:1700010.

29 Farkas L, Gauldie J, Voelkel NF, et al. Pulmonary hypertension and idiopathic pulmonary fibrosis. Am J Respir Cell Mol Biol 2011:45:1-15.

30 Ebina M, Shimizukawa M, Shibata N, et al. Heterogeneous increase in CD34-positive alveolar capillaries in idiopathic pulmonary fibrosis. Am I Respir Crit Care Med 2004;169:1203-8.

31 Renzoni EA, Walsh DA, Salmon M, et al. Interstitial vascularity in fibrosing alveolitis. Am J Respir Crit Care Med 2003;167:438-43.

32 Hubbard RB, Smith C, Le Jeune I, et al. The association between idiopathic pulmonary fibrosis and vascular disease: a population-based study. Am J Respir Crit Care Med 2008:178:1257-61.

33 Al-Bashir AK, Hillman GG. New DCE-MRI parameters to quantify the vascular changes induced by sunitinib treatment in renal carcinoma tumors. 2nd Middle East Conference on Biomedical Engineering; 17-20 Feb 2014, Qatar, 2014.

34 Hassoun PM, Nathan SD. Sildenafil for pulmonary hypertension complicating idiopathic pulmonary fibrosis: a rationale grounded in basic science. Eur Respir J 2016:47:1615-7.

35 Kolb M, Raghu G, Wells AU, et al. Nintedanib plus sildenafil in patients with idiopathic pulmonary fibrosis. N Engl J Med 2018;379:1722-31.

36 Wang JM, Robertson SH, Wang Z, et al. Using hyperpolarized ${ }^{129}$ Xe MRI to quantify regional gas transfer in idiopathic pulmonary fibrosis. Thorax 2018;73:21-8.

37 Ter-Karapetyan A, Triphan SMF, Jobst BJ, et al. Towards quantitative perfusion MRI of the lung in COPD: the problem of short-term repeatability. PLoS One 2018;13:e0208587

38 Østergaard L, Weisskoff RM, Chesler DA, et al. High resolution measurement of cerebral blood flow using intravascular tracer bolus passages. Part I: mathematical approach and statistical analysis. Magn Reson Med 1996;36:715-25.

39 Ohno Y, Hatabu H, Murase K, et al. Quantitative assessment of regional pulmonary perfusion in the entire lung using three-dimensional ultrafast dynamic contrastenhanced magnetic resonance imaging: preliminary experience in 40 subjects. J Magn Reson Imaging 2004;20:353-65.

40 Bossone E, D'Andrea A, D'Alto M, et al. Echocardiography in pulmonary arterial hypertension: from diagnosis to prognosis. J Am Soc Echocardiogr 2013;26:1-14. 